\title{
Aquablation for the Treatment of Benign Prostatic Hyperplasia in a Large Volume Prostate with an Intravesical Median Lobe
}

\author{
Eric M. Ghiraldi, DO, David Ambinder, BS, Young Son, BS, and Steven Sterious, MD, FACS
}

\begin{abstract}
Background: Aquablation is a relatively new minimally invasive technology for the treatment of benign prostatic hyperplasia (BPH) that has shown significant promise in its clinical efficacy and utility. Larger prostates or prostates with a median lobe are a common limitation to other minimally invasive treatment options. There is evidence that Aquablation maintains efficacy for larger prostates and prostates with an intravesical median lobe. Herein, we describe 3-month follow-up results for a patient who underwent Aquablation for a large prostate with a significant intravesical median lobe.

Case: Our patient is a 72-year-old man with lower urinary tract symptoms secondary to BPH refractory to pharmacotherapy. Patient underwent Aquablation of the prostate, which was $110 \mathrm{cc}$ in volume and had a large intravesical median lobe component. At 3-month follow-up patient had reduction in his AUA-SS from 21 to 12, improvement in uroflow from 8.2 to $16 \mathrm{~mL} / \mathrm{second}$, and improvement in postvoid residual from 90 to $13 \mathrm{~mL}$. Conclusion: Aquablation has been shown to be efficacious for the treatment of BPH in patients with large prostates and intravesical median lobes. This case report further demonstrates effective treatment for patients who fit this profile.
\end{abstract}

Keywords: Aquablation, benign prostatic hyperplasia, large prostate, robotics

\section{Introduction}

B ENIGN PROSTATIC HYPERPLASIA (BPH) is commonly seen in aging men. As BPH progresses, it can cause bladder outlet obstruction, which if not addressed can eventually lead to bladder decompensation, subsequent urinary retention, and upper urinary tract damage.

Initially, BPH with lower urinary tract symptoms (LUTS) is treated with medical therapy such as an $\alpha 1$-blocker or $5-\alpha$ reductase inhibitor. When medication fails to manage symptoms or cannot be tolerated because of side effects a number of surgical treatments can be offered. Unfortunately, many surgical treatments have limitations based on prostate size and the presence of a median lobe.

Contemporary literature shows a shift toward endoluminal minimally invasive techniques for bladder outlet obstruction, such as holmium laser enucleation of the prostate (HoLEP) ${ }^{1}$ and more recently Aquablation. ${ }^{2,3}$ Aquablation utilizes realtime ultrasonography with a robotically guided waterjet to ablate prostate tissue. Data regarding the efficacy and safety of Aquablation for large prostates, including prostates with intravesical median lobes, are limited to a single prospec- tive, multi-institutional, and international trial. The purpose of this report is to share our experience treating a patient with a large volume prostate and intravesical median lobe with Aquablation.

\section{Presentation of Case}

A 72-year-old man with a 5-year history of BPH medically managed with Finasteride. He was initially placed on dual pharmacotherapy, but had to stop Flomax secondary to retrograde ejaculation. His lower urinary tract symptoms included nocturia two to three times, urinary hesitancy, postvoid dribbling, and a previous episode of urinary retention. His American Urological Association Symptom Score (AUA-SS) was 21 with a mostly dissatisfied quality of life. Four years earlier, he had an elevated prostate specific antigen (PSA) and underwent a 12-core transrectal ultrasound-guided prostate biopsy that was negative for adenocarcinoma. His most recent PSA was 2.7 in January 2019 from 2.6 in December 2016, and 2.3 in November 2015, the time of his prostate biopsy. With persistent LUTS on Finasteride, he was interested in more definitive management.

Department of Urology, Albert Einstein Healthcare Network, Philadelphia, Pennsylvania, USA. 

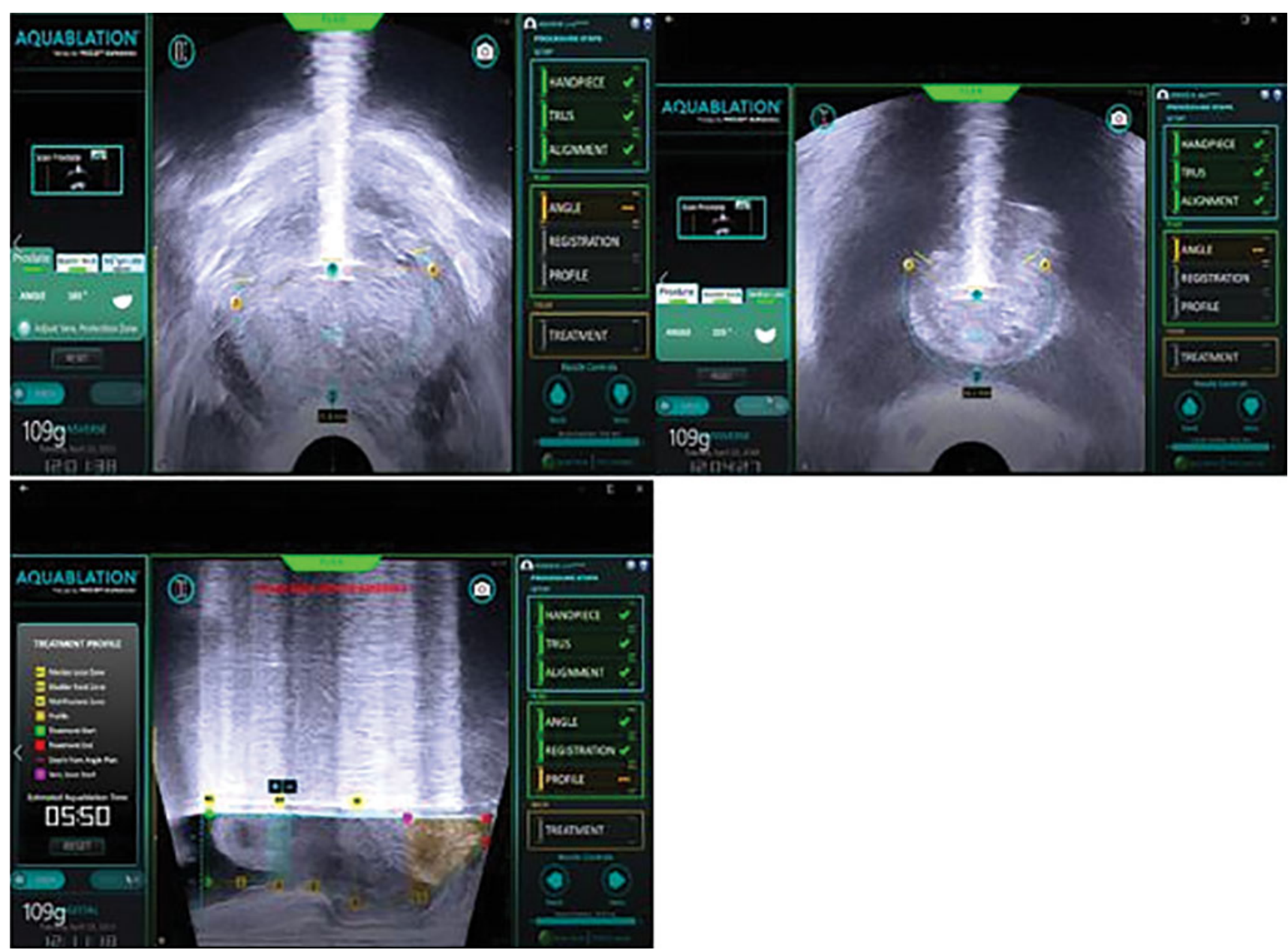

FIG. 1. Planning treatment metrics during Aquablation with markers adjusted for our treatment plan in the transverse plane (top left), for the median lobe (top right), and sagittal plane (bottom left).

Transabdominal ultrasound measured prostate volume to be $90 \mathrm{cc}$, giving him a PSA density of 0.06 (5.4/90 cc). Office cystoscopy revealed trilobar hypertrophy. Additional diagnostic work-up included a uroflow (Qmax) of $8.2 \mathrm{~mL} / \mathrm{second}$ and postvoid residual (PVR) of $90 \mathrm{~mL}$. Available treatment options offered to the patient included robotic simple prostatectomy, Aquablation, bipolar TURP, and UroLift. Our patient elected to proceed with Aquablation.

The procedure, as is done with the majority of cases performed at our institution, was done under spinal anesthesia. Figure 1 shows the treatment plan in the transverse and sagittal planes. The total time of resection was 5 minutes and 50 seconds. The bladder was then irrigated with a $22 \mathrm{~F}$ cystoscope and a $24 \mathrm{~F}$ three-way catheter was inserted and placed on traction with continuous bladder irrigation. Figure 2 shows the prostate in the sagittal plane before and after treatment. Our institution implements a specific postoperative protocol, which entails keeping the Foley on traction with continuous bladder irrigation for 4-6 hours that can be discontinued if the urine remains clear while off traction. In this case, the patient's Foley was taken off traction 6 hours after his procedure. Continuous bladder irrigation was continued overnight. On
POD \#1, urine remained clear after continuous bladder irrigation was discontinued. The patient passed his void trial with a PVR of $13 \mathrm{~mL}$. Hemoglobin trend was $14.9 \mathrm{gm} / \mathrm{dL}$ postoperatively to $15.0 \mathrm{~g} / \mathrm{dL}$ on postoperative day 1 .

At 3-month follow-up, our patient noted stronger urinary stream with resolution in lower urinary tract symptoms. AUA-SS improved to 12 from 21 with a "pleased" quality of life. On uroflow, patient demonstrated improvement in Qmax to 16 from $8.2 \mathrm{~mL} / \mathrm{second}$.

\section{Discussion}

As demonstrated by this case with follow-up results at 3 months, Aquablation can be a valuable tool in the urologists' armamentarium for the treatment of larger prostates. More importantly, precise treatment plan metrics allow for the treatment of prostates with intravesical median lobes. Our patient had resolution of lower urinary tract symptoms, improved uroflowmetry studies, and PVR volumes.

The majority of research focusing on the use of Aquablation for large prostates is derived from the Water II study, ${ }^{2,3}$ which was a multicenter international prospective trial that reported procedural outcomes in men with an 


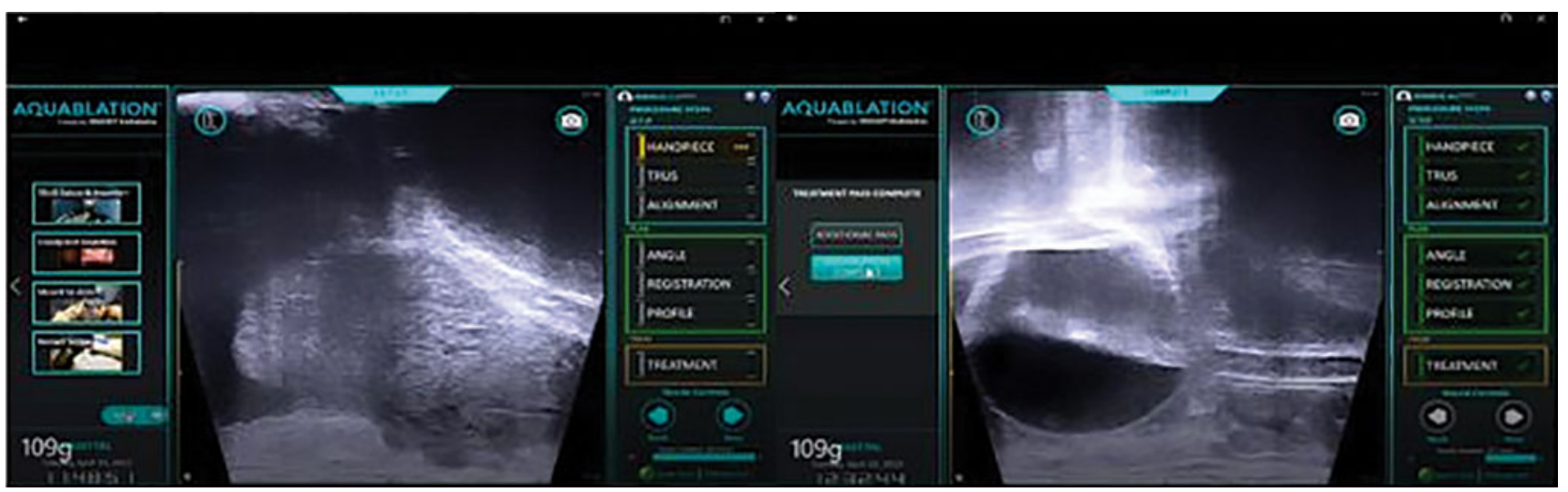

FIG. 2. Pretreatment (left) view of the prostate in sagittal plane demonstrating intravesical median lobe. Post-treatment (right) view of the prostate with Foley balloon placed on traction with $50 \mathrm{~mL}$ of sterile water in the balloon.

average prostate volume of $107 \mathrm{cc}^{2}$ Eighty-three percent of these patients had an intravesical median lobe with an average protrusion distance of $1.8 \mathrm{~cm}$ into the bladder. The study highlighted the ability of Aquablation to treat larger prostate volumes at short operating room times (mean, range; 55 minutes, 51-58 minutes) and short resection times (mean, range; 8 minutes, 3-17 minutes). Average length of hospital stay was 1.6 days. $^{2}$ One year follow-up data from the Water II demonstrates durable outcomes for International Prostate Symptom Score (IPSS), quality of life, Qmax, and PVR. Mean IPSS scores improved from 23.2 to 6.2. Mean quality of life scores improved from 4.6 to 1.3. Mean Qmax improved from 8.7 to $21.1 \mathrm{~mL} / \mathrm{second}$ and PVR decreased from $131 \mathrm{~mL}$ at baseline to $51 \mathrm{~mL}^{3}$.

HoLEP has gained popularity in recent years. Similar outcomes to simple prostatectomy (open, laparoscopy, robotassisted) have been shown regarding urinary flow rate (Qmax), IPSS, quality of life, and PVR. ${ }^{1}$ These comparable outcomes are obtained without having to make any incisions. A notable limitation of HoLEP is the learning curve required to master the surgical technique. A recent systematic review proposed that HoLEP had an acceptable learning curve of $\sim 25-50$ cases. ${ }^{4}$ Aquablation utilizes a user-friendly robotic software that is easy to learn after a single case. Furthermore, the software is combined with transrectal ultrasound guidance, ${ }^{2}$ which urologists are familiar with by the end of residency training.

One of the highlights of the Water II study compared with the Water I study was that electrocautery was not utilized after water jet ablation of prostate tissue. Overall a total of 16 bleeding complications occurred, with $6(5.9 \%)$ patients requiring blood transfusion during the index postprocedural hospitalization. An additional six patients required transfusion and/or cystoscopic fulguration for delayed bleeding. ${ }^{2}$ Our institution implements a specific protocol that starts intraoperatively immediately after tissue ablation. This entails immediate evacuation of blood clots in the bladder and evacuation of ablated tissue within the prostatic fossa. A $24 \mathrm{~F}$ hematuria catheter is then placed and secured on traction with $50 \mathrm{~mL}$ of sterile water in the Foley balloon. This process has been described in the Water I and Water II study. However, it is Foley catheter care after Aquablation that seems to vary. In the Water II study, traction was placed for an average of 18 hours, with a prostatic balloon for direct tamponade used in three patients for an average of 15 hours. From our early experience, prolonged traction makes patients uncomfortable once spinal anesthesia wears off, despite use of anticholinergics and belladonna-opium suppository. For this reason, we keep Foley traction on patients for 4-6 hours with continuous bladder irrigation. $\mathrm{Pa}-$ tients are then taken off traction and continuous bladder irrigation is continued until postoperative day 1. In our presented case, the patient was taken off traction in 46 hours after his procedure, taken off continuous bladder irrigation postoperative day 1 , and passed his void trial before discharge on postoperative day 1. Our patient's hemoglobin level postoperatively was $14.9 \mathrm{~g} / \mathrm{dL}$ and on postoperative day 1 it was $15.0 \mathrm{~g} / \mathrm{dL}$. The average decrease in hemoglobin in the Water II study was $2.9 \mathrm{~g} / \mathrm{dL}^{2}$.

\section{Conclusion}

Patients with BPH and LUTS that fail medical management have multiple surgical avenues, but larger prostates with median lobe involvement have less options. This case report details a patient with a large prostate and intravesical median lobe who has favorable perioperative and 3-month results after undergoing Aquablation.

\section{Disclosure Statement}

No competing financial interests exist.

\section{Funding Information}

No funding was received for this article.

\section{References}

1. Jones $\mathrm{P}$, Alzweri L, Rai BP, et al. Holmium laser enucleation versus simple prostatectomy for treating large prostates: Results of a systematic review and meta-analysis. Arab J Urol 2016;14:50-58.

2. Desai M, Bidair M, Bhojani N, et al. Water II (80-150 mL) procedural outcomes. BJU Int 2019;123:106-112. 
3. Bhojani N, Bidair M, Zorn KC, et al. Aquablation for benign prostatic hyperplasia in large prostates $(80-150 \mathrm{cc})$ : 1-year results. Urology 2019;129:1-7.

4. Kampantais S, Dimopoulos P, Tasleem A, et al. Assessing the Learning Curve of Holmium Laser Enucleation of Prostate (HoLEP). A systematic review. Urology 2018;120: 9-22.

Address correspondence to: Eric Ghiraldi, DO Department of Urology Albert Einstein Healthcare Network 1200 Tabor Road, 3rd Floor Philadelphia, PA 19141 USA

E-mail: ghiralde@einstein.edu

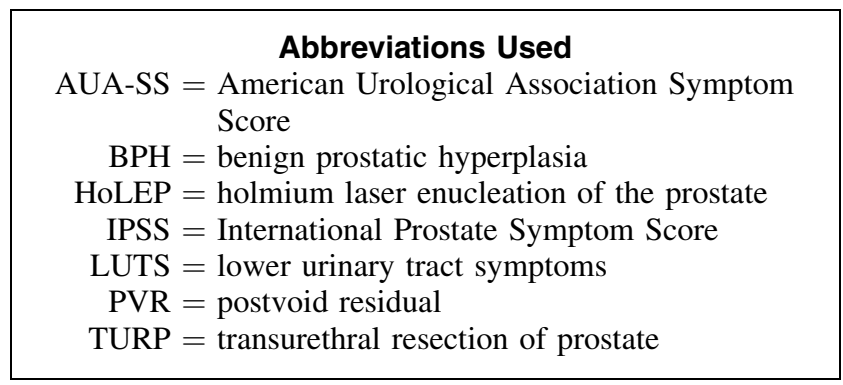

Cite this article as: Ghiraldi EM, Ambinder D, Son Y, Sterious S (2020) Aquablation for the treatment of benign prostatic hyperplasia in a large volume prostate with an intravesical median lobe, Journal of Endourology Case Reports 6:3, 110-113, DOI: 10.1089/cren.2019.0123. 